\title{
An explanation of the solar transition region
}

\author{
Philip Judge \\ High Altitude Observatory, National Center for Atmospheric Research ${ }^{1}$, P.O. Box 3000, \\ Boulder CO 8030\%-3000, USA
}

\begin{abstract}
Prompted by high resolution observations, I propose an explanation for the $40+$ year old problem of structure and energy balance in the solar transition region. The ingredients are simply cross-field diffusion of neutral atoms from cool threads extending into the corona, and the subsequent excitation, radiation and ionization of these atoms via electron impact. The processes occur whenever chromospheric plasma is adjacent to coronal plasma, and are efficient even when ion gyro-frequencies exceed collision frequencies. Cool threads - fibrils and spicules perhaps - grow slowly in thickness as a neutral, ionizing front expands across the magnetic field into coronal plasma. Radiative intensities estimated for $\mathrm{H} \mathrm{L} \alpha$ are within an order of magnitude of those observed, with no ad-hoc parameters - only thermal parameters and geometric considerations are needed. I speculate that the subsequent dynamics of the diffused material might also explain observed properties of trace elements.
\end{abstract}

Subject headings: Sun: atmosphere - Sun: chromosphere - Sun: transition region - Sun: corona - Sun: magnetic fields

\section{Introduction}

The upper transition region (henceforth, "TR") - plasma with electron temperatures in the range $2 \times 10^{5} \lesssim T_{e} \lesssim 10^{6} \mathrm{~K}$, is adequately described by field-aligned thermal conduction down from the corona. The lower TR $\left(10^{4} \mathrm{~K}<T_{e}<2 \times 10^{5} \mathrm{~K}\right)$ however, is not so easily understood (Gabriel 1976; Jordan 1980). Models dominated by field-aligned heat conduction produce too little emission from the lower TR by orders of magnitude, a problem already evident in work by Athay (1966). Neither could such models radiate away the downward directed conductive flux of $F_{\text {cond }} \sim 10^{6} \mathrm{ergs} \mathrm{cm}^{-2} \mathrm{~s}^{-1}$ (e.g. Jordan 1980; Athay 1981). Fontenla

\footnotetext{
${ }^{1}$ The National Center for Atmospheric Research is sponsored by the National Science Foundation
} 
et al. (2002 and earlier papers in the series), henceforth "FAL" showed that energy balance can be achieved through field-aligned (1D) diffusion of neutral hydrogen and helium atoms. The neutral atoms diffuse into hot regions, radiate away much of the coronal energy, and can reproduce the $\mathrm{H}$ and $\mathrm{He}$ line intensities.

The problem might be considered by some as solved, in principle. But there exists the serious and nagging problem of the peculiar spatial relationship between the observed corona, TR and chromosphere (Feldman 1983). Feldman and colleagues have since analyzed many observations, concluding that the lower TR is thermally disconnected from the corona (e.g. Feldman et al. 2001, and references therein). Yet Fontenla et al. (1990) declared that "The above [i.e. their] scenario explains why (as noted by Feldman 1983) the structure of the transition region is not clearly related to the structures in the corona". That the debate still rages is evidenced by advocates for "cool loop" models in which lower TR radiation originates from loops never reaching coronal temperatures and having negligible conduction (Patsourakos et al. 2007, and references therein, henceforth "PGV"). Here I propose a different scenario, prompted by new data and analyses which show that neither cool loops nor field-aligned processes adequately describe the $\mathrm{L} \alpha$ chromospheric network. I speculate that other TR lines might also be accounted for.

\section{A new scenario}

$\mathrm{L} \alpha$ network emission, at 0 '.3 resolution appears mostly as threads of relatively uniform intensity, of 5-10 Mm length and 1Mm diameter (PGV). PGV argued that "the different appearance the TR has in the quiet Sun [i.e. network] is suggesting that the bulk of its emission comes from structures other than the footpoints of hot loops". Convolved L $\alpha$ images from PGV appear to correspond to those seen in many other TR lines at lower resolution (e.g. Curdt et al. 2001). Judge and Centeno (2008) showed, using magnetic field measurements from Kitt Peak, that much of the network L $\alpha$ emission originates in long spicule-like structures lying along the lowest few Mm of magnetic field lines extending into the corona, but that plage emission may correspond to the thin footpoints as suspected by PGV and modeled by FAL. Even in plages, on sub-arcsecond scales, field-aligned threads of cool plasma (fibrils, spicules), extend into the low corona forming non-planar thermal interfaces between hot and cool plasma (Berger et al. 1999). Prompted by these data, I examine the diffusion of neutral particles into the corona, across magnetic fields (following a suggestion by Pietarila and Judge 2004).

Consider a straight cylinder of cool, partially ionized material embedded in a hot corona, of radius $r_{c}$. Length $l_{c} \gg r_{c}$ of the tube contains cool plasma in contact with the hot corona. 
The magnetic tube is of length $L \gg l_{c}$, mostly containing coronal plasma. Tube parameters are given in Table 1. Note that the neutral density greatly exceeds other densities. The chosen geometry is typical of values found by PGV, and thermal parameters are typical of the quiet Sun 1 . The plasma is assumed to be in a low plasma- $\beta$ regime.

\subsection{Initial diffusion, relaxation, radiation}

Imagine an injection of dense neutral material into the tube footpoint by some chromospheric process. The tube surface acts as a semi-permeable membrane. Neutral particles travel freely between collisions, but ions gyrate about magnetic field lines with gyro radii orders of magnitude smaller than mean free paths ("mfp"s, Table 21). Ions and electrons are essentially frozen to field lines, but neutrals can diffuse across field lines almost as efficiently as along them, and find themselves impacted by hot electrons and protons.

Table 2 lists time scales for kinetic processes for a "cool" hydrogen atom embedded in a hot corona of $T=10^{6} \mathrm{~K}$, using data from Allen (1973), Hansteen et al. (1997, henceforth HLH), and Gilbert et al. (2002). A hydrogen atom crossing the boundary encounters other diffusing hydrogen atoms and hot protons and electrons. Statistically, the first interaction is a collision with a coronal proton, involving the exchange of energy and ( $\sim 50 \%$ of the time) an electron (charge transfer, "CT"). Charge transfer yields an exchange of momentum $\left(180^{\circ}\right.$ change in direction) but little exchange of energy (e.g. Osterbrock 1961). The kinetic energy exchanged is $\sim \frac{3}{2} k T_{h}$, shared between them after two such collisions (I use subscript " $h$ " to denote hot and " $c$ " cool plasma). The CT cross section is roughly independent of energy, so the "warm" neutral atom has $\mathrm{a} \approx 1-e^{-1}=0.63$ probability of staying within the hot plasma. Assuming that it does so, after $\tau_{1 \kappa} \approx 8 \tau_{C T} \mathrm{~s}$ it becomes ionized by impact with a hot electron. Once free, the electron will not readily recombine with a proton (time scale $\left.\tau_{\kappa 1} \sim 3 \times 10^{5} \mathrm{~s}\right)$. At $T_{e}=T_{h}=10^{6} \mathrm{~K}$, the time $\tau_{12}$ needed for electron impact excitation of the $n=2$ levels and (rapid) emission of a $L \alpha$ photon is comparable to $\tau_{1 \kappa}$. Thus, of the ionized neutral atoms, $\approx 50 \%$ will have emitted a L $\alpha$ photon, the energy supplied by coronal electrons and protons. Because there are relatively few hot particles, their thermal energy limits the number of neutral atom ionizations and excitations.

From kinetic theory, the flux density of neutral hydrogen atoms initially crossing the boundary into the corona is $\frac{1}{4} n_{c} \bar{v}_{c} \sim 2 \times 10^{16}$ particles $\mathrm{s}^{-1} \mathrm{~cm}^{-2}$, where $\bar{v}=\sqrt{8 k T_{c} / \pi m}$. The kinetic energy per "hot" proton is $\frac{3}{2} k T_{h}$ which is shared roughly equally after two CT

\footnotetext{
${ }^{1}$ I do not adopt the higher temperatures of cool loops used by PGV, because here the corona and dynamics supplies all the energy for L $\alpha$ emission.
} 
collisions with a neutral, producing a population of $n_{h} \ll n_{c}$ "warm" neutrals with $T \sim T_{h} / 2$. After a few, say $m$ more collisions (time $m \tau_{C T}$ later), all the proton energies are the larger of $\sim \frac{3}{2} k T_{h} / 2^{m}$ and $\frac{3}{2} k T_{c}$, and $m n_{h}$ of the $n_{c}$ neutrals have suffered a proton impact. (A time of $n_{c} / n_{h}=100$ times $\tau_{C T} \approx 1 \mathrm{~s}$ is required before all neutrals have been impacted). The warm neutrals relax via collisions with the cool neutrals.

The initial electron evolution is largely determined by inelastic collisions with hydrogen: each hot electron typically has sufficient energy to excite and ionize 5 neutral hydrogen atoms, which takes $\sim 7 \tau_{1 \kappa} \sim 0.6 \mathrm{~s}$. (Electron-electron collision times are $\lesssim 0.05 \mathrm{~s}$ ). The electrons lose energy $\varepsilon=5 n_{h}(I+E) e$ per unit volume at the rate

$$
\frac{\varepsilon}{t} \gtrsim \frac{5 n_{h}(I+E) e}{7 \tau_{1 \kappa}} \approx 0.13 \mathrm{erg} \mathrm{cm}^{-3} \mathrm{~s}^{-1},
$$

leading to a cooling time of $\lesssim 0.4$ s. (A lower limit applies because tails of the Maxwellian distributions can increase excitation/ionization rates). Of this energy a fraction $\frac{E}{E+I}=\frac{3}{7}$ is emitted in $\mathrm{L} \alpha$. The radial flux density of $\mathrm{L} \alpha$ radiation from this neutral "sheath" is

$$
f \gtrsim \frac{3}{7} \frac{\varepsilon}{t} \Delta \equiv \frac{3}{7} \varepsilon v_{c}^{\text {diff }} \text { erg } \mathrm{cm}^{-2} \mathrm{~s}^{-1}
$$

where $\Delta$ is the sheath thickness at time $t\left(60 \tau_{C T}\right)$, and $v_{c}^{\text {diff }}=\Delta / t$ is the diffusion speed. For a random walk, $\Delta_{c} \approx \frac{1}{3} \sqrt{60} \tau_{C T} \bar{v}_{c} \approx 3.3 \times 10^{4} \mathrm{~cm}$, for warmed neutrals $\Delta_{w} \approx 1.8 \times 10^{5}$ $\mathrm{cm}\left(v_{c}^{\text {diff }}=0.57\right.$ and $v_{w}^{\text {diff }}=3.2 \mathrm{~km} / \mathrm{s}$ respectively; the factor $\frac{1}{3}$ accounts for the random direction of the "walk"). As a rough estimate, I take $v^{\text {diff }} \approx 3 v_{c}^{\text {diff }} \mathrm{km} / \mathrm{s}$ :

$$
f \approx \frac{3}{7} \varepsilon 3 v_{c}^{\text {diff }} \approx 5.6 \times 10^{3} \mathrm{erg}^{-2} \mathrm{~s}^{-1}
$$

Thus, $f$ is initially just a fixed fraction of the local coronal energy density multiplied by the diffusion speed. The specific intensity $I$ equals $f / \pi$ when the line is optically thick and all the radiation scatters away from the solar surface. (Photon mfps for $\mathrm{L} \alpha$ in the sheath are just $\left.10^{2} \mathrm{~cm}\right)$. This estimate of $I$ is a factor of $100-300$ below measured values of $(1.8-5.6) \times 10^{5}$ ergs $\mathrm{cm}^{-2} \mathrm{~s}^{-1} \mathrm{sr}^{-1}$ in active network threads (PGV) and 30 below average network intensities (Vernazza and Reeves 1978). But, as will be made clear below, this is an under-estimate. Similar estimates of intensities for $\mathrm{H} \mathrm{L} \beta$ and the $584 \AA$ line of He I, relative to L $\alpha$, are quite reasonable, recognizing that $\mathrm{L} \beta$ is optically thick across the sheath.

\subsection{A multi fluid calculation}

To examine the evolution at later times, multi-fluid equations for conservation of mass, momentum and energy were solved as functions of time and distance $x$ across the field lines 
following Schunk (1977) and HLH. Just electrons, protons and neutral hydrogen atoms were treated. Cartesian geometry is used because the diffusion region is much thinner than the tube. I assume that electrons are strongly tied to protons, so that their densities and fluid velocities are equal $\left(n_{e}=n_{p}, u_{e}=u_{p}\right.$ : charge and electrical currents are neglected). The conservation equations used for mass, momentum and energy density for the fluid of species $s$ are

$$
\begin{gathered}
\frac{\partial n_{s}}{\partial t}+\frac{\partial}{\partial x}\left\{n_{s} u_{s}+d_{s}^{n}\right\}=\frac{\delta n_{s}}{\delta t}, \\
m_{s} \frac{\partial n_{s} u_{s}}{\partial t}+\frac{\partial}{\partial x}\left\{m_{s} n_{s} u_{s}^{2}+p_{s}+d_{s}^{M}\right\}+F=\frac{\delta M_{s}}{\delta t}, \\
\frac{\partial E_{s}}{\partial t}+\frac{\partial}{\partial x}\left\{u\left(E_{s}+p_{s}\right)+d_{s}^{E}\right\}=\frac{\delta E_{s}}{\delta t}+Q-L .
\end{gathered}
$$

No conservation equation is used for the heat flux since here it is treated as $d_{s}^{E}$ using the mfp approximation. Above, $F$ is a body force term (gravity, Lorentz force for example), $E_{s}=\frac{3}{2} n_{s} k T_{s}+\frac{1}{2} m_{s} n_{s} u_{s}^{2}, p_{s}=n_{s} k T_{s}$, and the $\frac{\delta}{\delta t}$ are non-linear collisional terms. $Q$ and $L$ are the energy gains and losses respectively, where I adopt $Q=1.67 \times 10^{-25} n_{e} n_{p} e^{-T_{H} / 8000}$ erg $\mathrm{cm}^{3} \mathrm{~s}^{-1}$ to maintain a chromosphere against losses $L(\mathrm{HLH})$, and $L$ includes latent heat and $\mathrm{L} \alpha$ radiative losses computed explicitly from the collisional terms.

The diffusion terms $d_{s}$ (not included by HLH, except for the heat flux) require care especially for the dynamics of the proton fluid. For individual protons and electrons, the momentum equations are dominated by the Lorentz force. Their cross-field motion on timescales short compared with collision times is circular with frequency $\omega_{s}=e_{s} B / m_{s}$. On longer time scales the summed (fluid parcel) momenta can change only after a collision. The net effect of the Lorentz force is thus to limit the cross-field displacement of charged particles to a single gyro radius $r_{s}=\bar{v}_{s} / \omega_{s}$ in collision time $\tau_{s}$ instead of the collisional mean free path $\lambda_{s}=\bar{v}_{s} \tau_{s}$. Thus, a simple recipe for calculating cross-field transport via the fluid equations is to set both $F$ and $\frac{\partial p_{p}}{\partial x}$ terms to zero in the proton momentum equation, and modify the $d_{p}$ terms to account for the reduced displacements. Field-free diffusion is described by equations (4.41), (4.46) and (4.52) of Gombosi (1994):

$$
d_{s}^{n}=-\frac{1}{3} \lambda_{s} \frac{\partial}{\partial x}\left\{n_{s} \bar{v}_{s}\right\}, \quad d_{s}^{M}=-\frac{1}{3} \lambda_{s} \frac{\partial}{\partial x}\left\{m_{s} n_{s} u_{s} \bar{v}_{s}\right\}, \quad d_{s}^{E}=-\frac{\pi}{12} \lambda_{s} \frac{\partial}{\partial x}\left\{n_{s} m_{s} \bar{v}_{s}^{3}\right\}
$$

For charged particles, $\lambda_{s}$ must be replaced by $\lambda_{s}^{*}=\lambda_{s} /\left(1+\omega_{s} \tau_{s}\right)^{2}$ (following the above argument, see Braginskii 1965, eqs. 4.37, 4.40). Note that, written in terms of $T_{s}, d_{s}^{E}$ yields the widely used "Spitzer" thermal conductivity parallel to the field, and the ion-dominated conductivity perpendicular to the field. The net effect for $\omega_{s} \tau_{s} \gg 1$ is that only the neutral fluid diffuses efficiently across the field- the charged fluid evolves mostly via the collisional coupling to the neutrals (via $\delta M_{s} / \delta t$ ), and to a lesser degree to the small $d_{s}$ terms. 
The variables $\left(n_{s}, u_{s}, E_{s}\right)$ for electrons, protons and neutral $\mathrm{H}$ atoms, functions of $(t ; x)$, were initialized according to table 1. Only 7 variables were solved since it is assumed that $n_{e}=n_{p}$ and $u_{e}=u_{p}$. The equations were integrated in time using MacCormack's method to include the collisional terms (Griffiths and Higham 1999). For the first three points near $x=0$, the variables were held fixed to their initial values, maintaining the same chromospheric conditions there. Figure 1 shows conditions several seconds after the beginning of the diffusion process. Pressure gradients drive neutrals into the corona against friction forces, thus the diffusion speed, measured by tracking the steep temperature rise, is $\approx 0.8 \mathrm{~km} \mathrm{~s}^{-1}$, far below the thermal speed. The computed flux density of $\mathrm{L} \alpha$ is $\approx 5 \times 10^{4} \mathrm{ergs} \mathrm{cm}^{-2} \mathrm{~s}^{-1}$, and is roughly constant in time. It is some $10 \times$ higher than the simple kinetic result above, because of the nonlinear dynamics: (1) the densities become higher in the corona, (2) flow energy is converted to heat, (3) the L $\alpha$ losses/latent heat ratio is higher (the photons are created at electron temperatures lower than the initial coronal temperature). $I$ is computed to be just a factor of 10-30 below observed active network thread intensities, and 3 below average network intensities.

A calculation with twice the coronal density, more appropriate for active network, yields smaller diffusion speeds and L $\alpha$ fluxes which are just 1.7 times higher. EUV/X-ray coronal intensities scale with (density) ${ }^{2}$, and so would be a factor of four brighter. This non-linear relationship is an important property of the calculations.

\section{Discussion, speculations}

Based upon observations of spicules and other fine, thread-like structures on the solar disk, it is clear that non-planar thermal interfaces exist at the base of the corona, and that the morphology of the TR emission from such interfaces cannot be explained by field-aligned particle transport at the base of coronal loops, in contrast to the claims by Fontenla et al. (1990). The picture proposed here uses unspecified mechanisms in the chromosphere to maintain a reservoir of cool mostly neutral plasma directly adjacent to hot coronal plasma. The cylindrical geometry, inspired by observations, presents a large surface area (per unit volume) of contact between cool and hot plasma. The chromosphere supplies mass via neutral diffusion across the surface to a thermal boundary layer, and the corona supplies energy to the neutral particles. The originally neutral particles drain energy from the corona by latent heat of ionization and by inelastic collisions leading to strong $\mathrm{L} \alpha$ emission. The diffusing layer propagates outwards, emitting radiation like the boundary of a wild fire 2 , into the

\footnotetext{
${ }^{2}$ Secchi in 1877 described the chromosphere as a "burning prairie", but in a different sense.
} 
corona until either the supply of neutral mass or coronal energy dries up. The present proposal is related to models invoking cross-field heat conduction (Rabin and Moore 1984; Athay 1990). This effect is included here (via $d_{s}^{E}$ ), but it is far less efficient at moving heat to cool plasma than diffusion is at moving neutral atoms to the coronal heat.

The calculations presented here fall short of accounting for the large radiative flux of $\mathrm{L} \alpha$, by factors of $\approx 10$. However, the calculations miss important additional sources of energy in the corona: thermal and gravitational potential energy. The cool threads extend only a few $\mathrm{Mm}$ into the corona, and form just the lower parts of a much larger coronal structure. The diffused cool material is thus subject to parallel transport (heat conduction, diffusion) which will transfer heat from the overlying coronal plasma to the diffused material. Spicules formed by ejection from the chromosphere will have their entire length exposed to this energy flux, because the lowest parts of the spicules diffuse first into the corona- the diffusion fronts are not exactly parallel to field lines. Coronal plasma along connected field lines contains $L \frac{3}{2} n k T$ erg $\mathrm{cm}^{-2}$, where $L$ is the pressure scale height $(\sim 50 \mathrm{Mm})$ or loop length. Since $L \gg l_{c}$ the energy available for $\mathrm{L} \alpha$ radiation would be $L / l_{c} \geq 10 \times$ larger than computed above, more if the tubes expand with height. I speculate that cross-field diffusion and subsequent parallel conduction might bring theoretical and observed intensities values into agreement. The time needed to conduct this energy must lie between the electron sound speed $c_{e}$ as $L / c_{e} \sim 13 \mathrm{~s}$, and $\sim 10^{3} \mathrm{~s}$, an upper limit obtained from the thermal energy divided by the conductive flux for a uniform temperature gradient. Gravitational potential energy might contribute to the heating and dynamics of the sheath as the diffused material cools the corona and adds mass, such that vertical pressure balance no longer is expected. It may be that larger red-shifts would be expected where magnetic fields are more vertical, i.e. directly over the magnetic network. This expectation is not in disagreement with results found by McIntosh et al. (2007). However, little more can be said without solving the $2 \mathrm{D}$ multi-fluid conservation equations including parallel heat conduction and cross-field diffusion, beyond the scope of this letter. Such calculations will also show if the emission lines of trace species (ions of carbon, oxygen etc. in the TR) can be explained.

Cool threads are observed in different coronal environments (PGV)- their intensities appear to vary relatively little compared with the embedding coronal intensities. This fact is part of Feldman's (1983) claim that TR emission is energetically disconnected from the corona. The calculations presented here indeed produce a non-linear relationship between $\mathrm{L} \alpha$ and coronal brightness. The $\mathrm{L} \alpha$ intensities scale with the local coronal energy density and with the diffusion speed. But the EUV and X-ray radiation emitted by the corona itself vary with density ${ }^{2}$ and peaked functions of temperature along lines of sight different from the direction of field lines into the sheath. The scenario might therefore explain most of the observed puzzling facets noted by Feldman and colleagues, yet still maintain a strong 
energetic link between the corona and TR, and thereby resolve a long-standing debate (see the different perspectives of Feldman et al. 2001 and Wikstøl et al. 1998, for example).

To see if the scenario survives scrutiny, more observations of chromospheric fine structure and its relation with the corona and TR would be as important as numerical modeling work. I am grateful to Tom Holzer, Scott McIntosh and the referee for comments.

\section{REFERENCES}

Allen, C. W.: 1973, Astrophysical Quantities, Athlone Press, Univ. London

Athay, R. G.: 1966, Astrophys. J. 145, 784

Athay, R. G.: 1981, Astrophys. J. 249, 340

Athay, R. G.: 1990, Astrophys. J. 362, 364

Berger, T. E., De Pontieu, B., Schrijver, C. J., and Title, A. M.: 1999, Astrophys. J. Lett. 519, L97

Braginskii, S. I.: 1965, Reviews of Plasma Physics. 1, 205

Curdt, W., Brekke, P., Feldman, U., Wilhelm, K., Dwivedi, B. N., Schühle, U., and Lemaire, P.: 2001, Astron. Astrophys. 375, 591

Feldman, U.: 1983, Astrophys. J. 275, 367

Feldman, U., Dammasch, I. E., and Wilhelm, K.: 2001, Astrophys. J. 558, 423

Fontenla, J. M., Avrett, E. H., and Loeser, R.: 1990, Astrophys. J. 355, 700

Fontenla, J. M., Avrett, E. H., and Loeser, R.: 2002, Astrophys. J. 572, 636 (FAL)

Gabriel, A.: 1976, Phil Trans. Royal Soc. Lond. 281, 339

Gilbert, H. R., Hansteen, V. H., and Holzer, T. E.: 2002, Astrophys. J. 577, 464

Gombosi, T. I.: 1994, Gaskinetic Theory, Cambridge University Press, Cambridge, England

Griffiths, D. and Higham, D.: 1999, MacCormack's method for advection-reaction equations, Technical report, Department of Mathematics, University of Strathclyde

Hansteen, V., Leer, E., and Holzer, T.: 1997, Astrophys. J. 482, 498 (HLH) 
Jordan, C.: 1980, Astron. Astrophys. 86, 355

Judge, P. G. and Centeno, R.: 2008, Astrophys. J. in press

McIntosh, S. W., Davey, A. R., Hassler, D. M., Armstrong, J. D., Curdt, W., Wilhelm, K., and Lin, G.: 2007, Astrophys. J. 654, 650

Osterbrock, D. E.: 1961, Astrophys. J. 134, 347

Patsourakos, S., Gouttebroze, P., and Vourlidas, A.: 2007, Astrophys. J. 664, 1214 (PGV)

Pietarila, A. and Judge, P. G.: 2004, Astrophys. J. 606, 1239

Rabin, D. and Moore, R.: 1984, Astrophys. J. 285, 359

Schunk, R. W.: 1977, Reviews of Geophysics and Space Physics 15, 429

Vernazza, J. E. and Reeves, E. M.: 1978, Astrophys. J. Suppl. Ser. 37, 485

Wikstøl, Ø., Judge, P. G., and Hansteen, V.: 1998, Astrophys. J. 501, 895 
Table 1. Tube properties

\begin{tabular}{|c|c|c|}
\hline Quantity & $\begin{array}{l}\text { Inside } \\
(\mathrm{cool})\end{array}$ & $\begin{array}{l}\text { Outside } \\
\text { (hot) }\end{array}$ \\
\hline radius $r_{c} \mathrm{~cm}$ & $5 \times 10^{7}$ & \\
\hline length $l_{c} \mathrm{~cm}$ & $5 \times 10^{8}$ & $\geq 10 l_{c}$ \\
\hline$T \mathrm{~K}$ & $10^{4}$ & $10^{6}$ \\
\hline$n_{H} \mathrm{~cm}^{-3}$ & $8 \times 10^{10}$ & $\approx 0$ \\
\hline$n_{p} \mathrm{~cm}^{-3}$ & $n_{H} / 40$ & $4 \times 10^{8}$ \\
\hline$n_{e} \mathrm{~cm}^{-3}$ & $n_{H} / 40$ & $4 \times 10^{8}$ \\
\hline$p$ dyn $\mathrm{cm}^{-2}$ & 0.11 & 0.11 \\
\hline Magnetic field strength $B \mathrm{G}$ & 10 & 10 \\
\hline$B^{2} / 8 \pi$ dyn $\mathrm{cm}^{-2}$ & 3.8 & 3.8 \\
\hline
\end{tabular}


Table 2. Plasma conditions

\begin{tabular}{|c|c|c|c|c|}
\hline Quantity & Units & & scaling & notes \\
\hline \multicolumn{5}{|l|}{ Initial corona } \\
\hline$n_{h}$ & $\mathrm{~cm}^{-3}$ & $8.0 \times 10^{8}$ & & \\
\hline$n_{p}, n_{e}$ & $\mathrm{~cm}^{-3}$ & $4.0 \times 10^{8}$ & & \\
\hline$p$ & $\mathrm{~cm}^{-3}$ & $1.1 \times 10^{-1}$ & & \\
\hline$B$ & G & 10 & & \\
\hline$\beta$ & & $2.8 \times 10^{-2}$ & & \\
\hline$\omega_{p}$ & $\mathrm{~s}^{-1}$ & $9.6 \times 10^{4}$ & & \\
\hline$r_{\text {gyro }}$ & $\mathrm{km}$ & $1.5 \times 10^{-3}$ & & \\
\hline$\tau_{p p}$ & s & 1.6 & $n_{p}^{-1} T^{+3 / 2}$ & \\
\hline$\omega_{p} \tau_{p p}$ & & $1.5 \times 10^{5}$ & & \\
\hline & $\mathrm{s}$ & $5.0 \times 10^{-2}$ & $n_{e}^{-1} T^{3 / 2}$ & $\begin{array}{l}\tau_{e e} \mathrm{~s} \\
\text { chromospheric tube }\end{array}$ \\
\hline $\begin{array}{l}\text { chromospheric ti } \\
\quad T_{c}\end{array}$ & $\begin{array}{l}\text { be } \\
\text { K }\end{array}$ & $8.0 \times 10^{3}$ & & \\
\hline $\bar{v}$ & $\mathrm{~km} \mathrm{~s}^{-1}$ & 13 & $T^{1 / 2}$ & \\
\hline$n_{c}$ & $\mathrm{~cm}^{-3}$ & $10^{11}$ & & \\
\hline$\tau_{n n}$ & $\mathrm{~s}$ & $1.4 \times 10^{-2}$ & $n_{n}^{-1} T^{-1 / 2}$ & \\
\hline \multicolumn{5}{|c|}{ hot protons impacting hydrogen atoms } \\
\hline$\tau_{p n}(C T)$ & $\mathrm{s}$ & $1.0 \times 10^{-2}$ & $n_{p}^{-1} T^{-1 / 2}$ & $" C T "=$ charge transfer \\
\hline $\mathrm{H}$ atom $\mathrm{mfp}$ & $\mathrm{km}$ & $6.5 \times 10^{-2}$ & $n_{p}^{-1}$ & \\
\hline \multicolumn{5}{|c|}{ cool hydrogen atoms impacting protons ${ }^{p}$} \\
\hline$\tau_{n p}(C T)$ & s & $8.0 \times 10^{-5}$ & $n_{n}^{-1} T^{-1 / 2}$ & \\
\hline proton $\mathrm{mfp}$ & $\mathrm{km}$ & $5.8 \times 10^{-3}$ & $n_{n}^{-1}$ & \\
\hline \multicolumn{5}{|c|}{$\begin{array}{cc}\omega_{p} \tau_{n p} & 7.7 \\
\text { hot electrons impacting H atoms }\end{array}$} \\
\hline$\tau_{12}$ & $\mathrm{~s}$ & $9.5 \times 10^{-2}$ & $n_{e}^{-1} T_{e}^{-1 / 2} e^{10.2 e / k T_{e}}$ & excitation of $n=2$ level \\
\hline$\tau_{1 k}$ & s & $8.2 \times 10^{-2}$ & $n_{e}^{-1} T_{e}^{-1 / 2} e^{13.6 e / k T_{e}}$ & ionization \\
\hline$\tau_{k 1}$ & $\mathrm{~s}$ & $4.0 \times 10^{5}$ & $n_{e}^{-1} T_{e}^{+1 / 2}$ & radiative recombination \\
\hline
\end{tabular}


Note. - $\tau_{a b}$ refers to the time taken for a particle of type $b$ to be impacted by a sea of particles of type $a$, except where noted. 

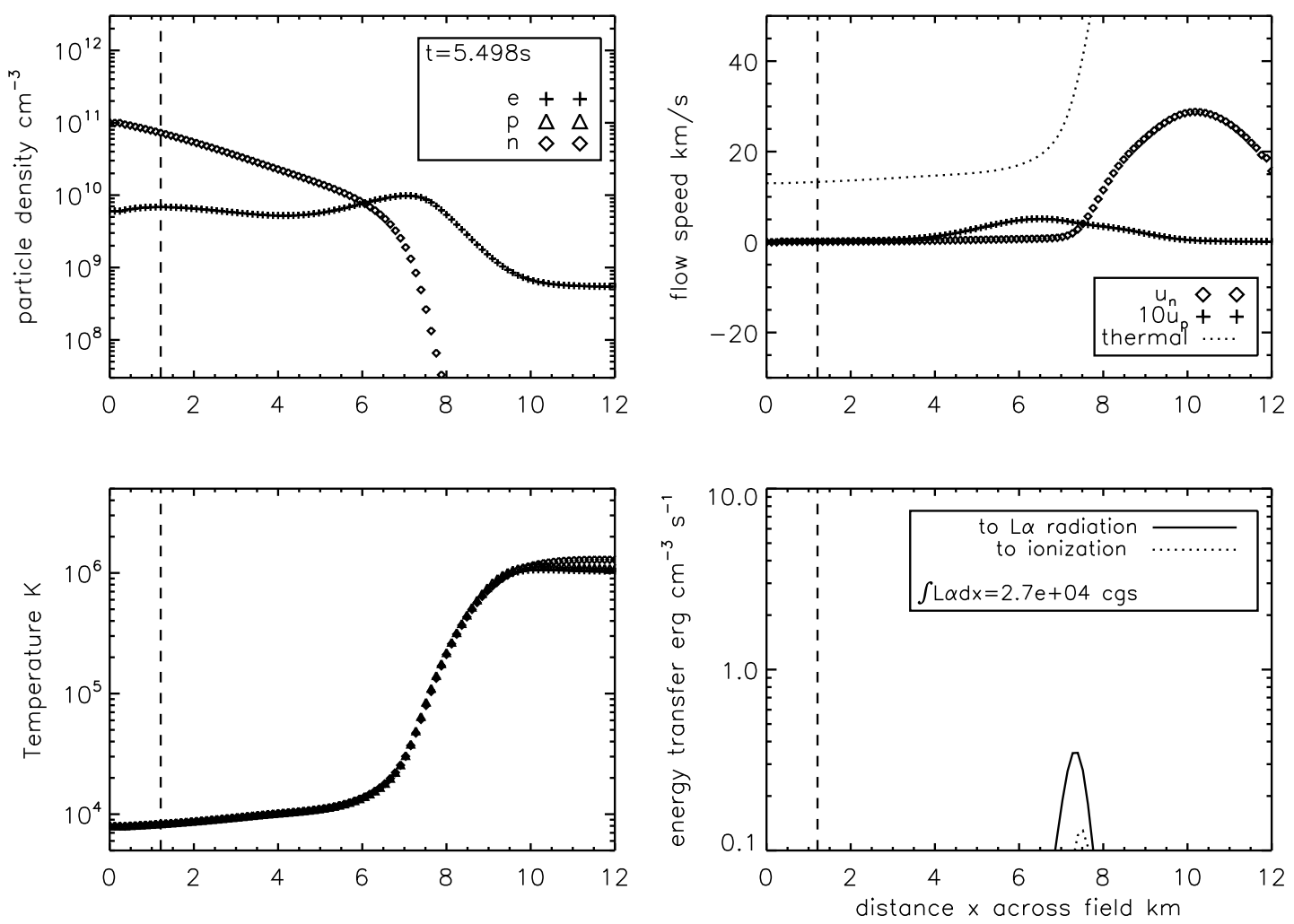

Fig. 1. - Conditions a few seconds after hydrogen is allowed to diffuse across field lines into coronal plasma. The abscissa is distance $x$ across the field lines, the initial cool flux tube extends from zero to the dashed line. 